\title{
Erratum
}

\section{The size of random bucket trees via urn models}

\section{Hosam M. Mahmoud}

The George Washington University, Department of Statistics, Washington, DC 20052, USA (e-mail: hosam@gwu.edu)

Published online: 14 October 2004 - (C) Springer-Verlag 2004

Acta Informatica 38, 813-838 (2002)

The Equation (2) in [1] is not exact as claimed. It is only approximate, and there are error terms that seem hard to determine. However, it gives a basis for a good heuristic based on the observation that the interarrival time $t_{n}$ is highly concentrated around its mean. The relation (3) is therefore approximate, though it can be proved rigorously in several special cases, and is observed experimentally in all the cases. The results of the heuristic agree closely with extensive simulation. The companion paper [2] explains the working of the heuristic in a broader class of urns.

Acknowledgements. My understanding of the heuristics in depoissonization has been considerably enhanced by discussions with Professor Svante Janson.

\section{References}

1. Mahmoud, H. (2002) The size of random bucket trees via urn models. Acta Informatica 38, 813-838

2. Mahmoud, H. (2004) Random sprouts as Internet models, and Pólya processes. Acta Informatica DOI 10.1007/s00236-004-146-y

The online version of the original article can be found at http://dx.doi.org/10.1007/s00236-002-0096-1 